\title{
Partitioned Hermitian Matrices*
}

\author{
Russell Merris \\ Institute for Basic Standards, National Bureau of Standards, Washington, D.C., 20234 \\ (December 12, 1969)
}

A class of Cauchy-Schwarz type inequalities for partitioned hermitian matrices is presented.

Key words: Generalized matrix function; positive semi-definite hermitian matrix.

Let $M_{n}^{r}$ denote the set of $r \times n$ matrices over the complex numbers. Write $M_{n}$ for $M_{n}^{n}$. Let $G$ be a subgroup of $S_{m l}$, the symmetric group on $m l$ symbols. Suppose $\lambda$ is a character of degree 1 on $G$. If $X=\left(x_{i j}\right) \epsilon M_{m l}$, the generalized matrix function of $X$ is

$$
d(X)=\sum_{\sigma \in G} \lambda(\sigma) \prod_{t=1}^{m l} x_{t \sigma(t)}
$$

Let $f: M_{p} \rightarrow M_{m}$ be any function. Then $f$ induces a function $\varphi_{f}: M_{p q} \rightarrow M_{m q}$ as follows:

$$
\varphi_{f}(X)=\left(f\left(X_{s t}\right)\right)
$$

where $X=\left(X_{s t}\right)$ is a block matrix in which $X_{s t}$ is a $p \times p$ submatrix of $X, 1 \leqslant s, t \leqslant q$.

Let $A_{1}, \ldots, A_{k} \in M_{p l}^{r}$. Let $H$ be the block matrix

$$
H=\left(A_{i}^{*} A_{j}\right) \epsilon M_{p k l}
$$

( $A_{i}^{*}$ is the conjugate transpose of $A_{i}$.)

Lemma. The matrix $\mathrm{H}$ is positive semi-definite hermitian (psdh).

Proof:

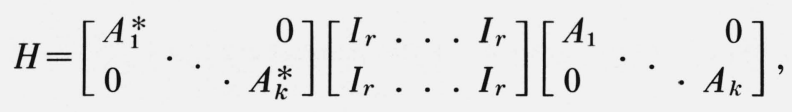

where $I_{r} \in M_{r}$ is the identity matrix.

(Observe that in general the block matrix whose $i$, jth block is $A_{j}^{*} A_{i}$ is not $p s d h$. Take

$$
\left.A_{1}=\left[\begin{array}{ll}
1 & 1 \\
0 & 1
\end{array}\right], A_{2}=I_{2} .\right)
$$

Suppose $f$ is such that $\varphi_{f}(H)$ is $p s d h$. We may write

$$
\varphi_{f}(H)=\left(\varphi_{f}\left(A_{i}^{*} A_{j}\right)\right)
$$

*This work was done (1969-1970) while the author was a National Academy of Sciences-National Research Council Postdoctoral Research Associate at the National Bureau of Standards, Washington, D.C. 20234. 
Now, by a trivial consequence of a result of Marcus and Katz [4, Theorem 3], ${ }^{1}$ the block matrix

$$
\left(d\left(\varphi_{f}\left(A_{i}^{*} A_{j}\right)\right)\right)
$$

is $p s d h$. Taking the determinant of the leading $2 \times 2$ principal submatrix, one obtains the main result:

THEOREM.

$$
\mathrm{d} \varphi_{\mathrm{f}}\left(\mathrm{A}_{1}^{*} A_{1}\right) d \varphi_{\mathrm{f}}\left(\mathrm{A}_{2}^{*} A_{2}\right) \geqslant \mathrm{d} \varphi_{\mathrm{f}}\left(A_{1}^{*} A_{2}\right) \mathrm{d} \varphi_{\mathrm{f}}\left(A_{2}^{*} A_{1}\right)
$$

One easily sees that a string of further inequalities is available by taking any generalized matrix function of any of the leading principal submatrices.

Some functions, $f$, which have the property that $\varphi_{f}$ sends psdh matrices to psdh matrices have been discovered. A partial list follows. Let $X \epsilon M_{p}$.

1. Let $f(X)=X$. Then the theorem reduces to the well known result $([9$, p. 168] or $[5$, p. 323])

$$
d\left(A^{*} A\right) d\left(B^{*} B\right) \geqslant\left|d\left(A^{*} B\right)\right|^{2} .
$$

When $p=l=1$, this is the Cauchy-Schwarz inequality.

2. If $p=1$, let $f(x)=x^{r}$, where $r$ is a positive integer. Then Schur proved $f$ has the required property [8]. Löwner [2] extended this to cover the case for any real number at least 1 .

3. If $p=1$, let $f(x)=|x|^{2}[3]$.

4. Let $S$ be a subgroup of $S_{p}$ and $\chi$ a character on $S$, let

$$
f(X)=\sum_{\sigma \in S} \chi(\sigma) \sum_{t=1}^{p} x_{t \sigma(t)}
$$

5. For $l=1, k=2$, let $f(X)=$ trace $\left(X^{2}\right)$, [6].

6. For a given symmetry class of tensors arising from a group and a character of degree 1 , let $f(X)=K(X)$ be the associated matrix, or let $f(X)$ be any generalized matrix function of $X[4$, Theorem 3]. (For a discussion of these terms, see [9], [6], [10].)

7. Let $\alpha_{1}^{2}(X), \ldots, \alpha_{p}^{2}(X)$ be the squares of the singular values of $X$. Let

$$
f(X)=\operatorname{trace} K\left(\operatorname{diag}\left(\alpha_{1}^{2}(X), \ldots, \alpha_{p}^{2}(X)\right)\right)
$$

be the Schur function of the squares of the singular values of the matrix $X[6]$. We may take

$$
f(X)=E_{s}\left(\lambda_{1}(X), \ldots, \lambda_{p}(X)\right)
$$

to be the $s$ th elementary symmetric function of the eigenvalues of $X[1]$.

The author thanks Morris Newman for shortening the proof of the lemma.

\section{References}

[1] de Pillis, J., Transformations on partioned matrices, Duke Math. J. 36, 511-515 (1969).

[2] Löwner, Charles, Some Theorems on Positive Matrices (unpublished).

[3] Marcus, Marvin, and Khan, Nisar A., A note on the Hadamard product, Canad. Math. Bull. 2, 81-83 (1959).

[4] Marcus, Marvin, and Katz, Susan M., Matrices of Schur functions, Duke Math. J. 36, 343-352 (1969).

[5] Marcus, Marvin, and Minc, Henryk, Generalized matrix functions, Trans. Amer. Math. Soc. 116, 316-329 (1965).

[6] Marcus, Marvin, and Watkins, William, Partitioned hermitian matrices, Duke Math. J. (submitted).

[7] Merris, Russell, Trace functions 1, Duke Math. J. (submitted).

[8] Mirsky, L., Ān Introduction to Linear Algebra, 421 (Clarendon Press, Oxford, England 1955).

[9] Shisha, Oved (ed.), Inequalities, 163-176 (Academic Press, 1967).

[10] Wedderburn, J. H. M., Lectures on Matrices, Amer. Math. Soc., Colloq. Publ. vol. 17, New York (1934).

${ }^{1}$ Figures in brackets indicate the literature references at the end of this paper. 